\title{
1 Complete sequence and genomic annotation of Carrot torradovirus
}

\section{1 (CaTV1)}

3 Zuriñe Rozado-Aguirre ${ }^{1,2}$, Ian Adams ${ }^{1}$, Adrian Fox ${ }^{1}$, Matthew Dickinson ${ }^{2}$, and Neil Boonham ${ }^{1,3}$

$4 \quad{ }^{1}$ Plant Protection Programme, Fera, Sand Hutton, York, YO41 1LZ, UK

5 'University of Nottingham, Sutton Bonington, Leicestershire, LE12 5RD, UK

$6{ }^{3}$ IAFRI, Newcastle University, Newcastle upon Tyne, NE1 7RU

7

8 Corresponding author: zurine.rozado@fera.co.uk

\section{Abstract}

Carrot torradovirus 1 (CaTV1) is a new member of the Torradovirus genus within the family Secoviridae. CaTV1 genome sequences were obtained from a previous Next Generation Sequencing (NGS) study and were compared to other members and tentative new members of the genus. The virus is comprised of a bipartite genome and RACE was used to amplify and sequence each end of RNA1 and RNA2. As a result RNA1 and RNA2 are estimated as containing 6944 and 4995 nucleotides respectively, with RNA1 encoding the proteins involved in virus replication, and RNA2 encoding the encapsidation and movement proteins. Sequence comparisons showed that CaTV1 clustered within the non-tomato infecting torradoviruses and is most similar to Motherwort yellow mottle virus (MYMoV). The nucleotide identities of the Pro-Pol and coat protein regions were below the criteria established by the ICTV for demarcating species, confirming that CaTV1 should be classified as a new species within the Torradovirus genus.

\section{Introduction}

Carrot torradovirus 1 (CaTV1) was an incidental finding discovered in a Next Generation Sequencing (NGS) study seeking to elucidate the causes of internal necrosis in carrots (Daucus carota) in the UK 
27 [1]. CaTV1 has been recently reported in carrot leaves collected in the Southwest of France, indicating its presence also outside the UK [2]. The virus is similar to members of the Torradovirus genus, first described in 2007 to place two new viruses affecting tomato crops, Tomato torrado virus (ToTV) and Tomato marchitez virus (ToMarV) [3, 4]. Later more viruses affecting different crops have been added to the genus, including, tomato chocolate virus (ToChV), Tomato chocolate spot virus (ToChSV), Tomato necrotic dwarf virus (ToNDV), Lettuce necrotic leaf curl virus (LNLCV), Motherwort yellow mottle virus (MYMoV), Cassava torrado-like virus (CsTLV) and Squash chlorotic leaf spot virus (SCLSV) [5-11]. Torradoviruses are considered members of the Secoviridae family within the order Picornavirales [12] and previous sequence comparisons within this genus established two different clades for tomato-infecting (TI) and non-tomato infecting (NTI) members [13].

Although CaTV1 was a sequencing finding using NGS, the sequence of the virus was not fully described. In this study we complete the characterization of the genome of CaTV1, including completion of the $3^{\prime}$ and 5'ends of both RNA fragments using RACE, annotation of the genome identifying the location of translational features, and establishing the similarities between this and other members of the Torradovirus genus.

\section{Completion of the CaTV1 genome}

The $3^{\prime}$ and $5^{\prime}$ ends of both RNA1 and RNA2 were amplified using the SMARTer RACE cDNA amplification kit (Clontech) according to the manufacturer's protocols. The 3' and 5' PCR products were analysed by direct sequencing. Results indicated that the 5' UTR and 3' UTR regions of RNA1 were 127 and 240 nt long respectively, and for RNA2, were 611 nt (5' UTR) and 327 (3' UTR) in length. Completed sequences were deposited in GenBank with accession numbers KF533719.2 and KF533720.2 for RNA1 and RNA2 respectively.

\section{CaTV1 genome organization}

CaTV1 - RNA1 
As a member of the Secoviridae family, RNA1 is likely to code for a single ORF translated into a polyprotein which is then likely to be processed by serine-like proteases into mature proteins [12]. Following analysis of RNA1, one predicted ORF (RNA1-ORF1) was identified (6944 nts), encoding a putative polyprotein of 2192 amino acids (aa) with a molecular mass of $249 \mathrm{kDa}$. The translational start (AUG) and stop (UAA) codons were found at nucleotide positions 127-129 and 6703-6705 respectively (Figure 1).

The complete nucleotide sequence was compared to other sequences available in GenBank using BlastN, revealing $71 \%$ identity to LNLCV (KC855266) and ToMarV (KT756874), $69 \%$ to ToChSV (GQ305131) and ToTV (KM091449) and 68 \% to MYMoV (KM855266) and ToNDV (KC999058), all members of the Torradovirus genus.

Identification of protein motifs were made based on previous characterization of ToTV and ToMarV [3, 4]. The polyprotein contains the conserved Hel-Pro-Pol replication block typical of picorna-like viruses (nt positions 401-1516). Comparison of the aa sequence of the Pro-Pol region (1072-1516), limited by the "CG" motif of the 3C-like proteinase and the "GDD" motif of the RNA-dependent RNA polymerase (RdRp), suggested levels of similarity lower than those specified in the species demarcation criteria (<80\%) established in the ninth ICTV report [14]. This conserved domain is typically used to determine differences among different picornavirales members. Pairwise comparisons using the Hel-Pro-Pol protein sequences with other torradoviruses, showed that CaTV1 shares $58.5 \%, 59.5 \%$ and $41.2 \%$ of the sequence with LNLCV, MYMoY and SCLSV respectively. Lower levels were found when the same region was compared to tomato-infecting torradoviruses. Typical helicase motifs (type III helicase), A (GKT), B (DD) and C (N) were found at positions 410, 456 and 507 respectively. The region, between aa $401-508$, shares levels of identity up to $84 \%$ with the corresponding region of LNLCV and $82.9 \%$ with MYMoV, while lower levels of identity were found in the tomato-infecting torradoviruses (46.4\% ToTV and $50 \%$ ToMarV). A histidine residue in the putative protease substrate binding pocket is located at aa position 1075 and is required for proteolytic processing in members of the Secoviridae family. Typical RdRp motifs (I-VII) were found between aminoacid positions 1311-1596 [15]. Pairwise comparison revealed that the closest amino acid identities in the RdRp domain were found with LNLCV (75.1\%) and MYMoV and ToChV (70.5 $\%)$. 
To determine the relationship between CaTV1 and other viruses of the family, a neighbour-joining phylogenetic tree with 500 bootstrap replications was constructed with the RdRp region using MEGA6 and ClustalX (figure 2a). The analysis revealed different clades for TI and NTI members confirming previous results described [13]. It also indicates differences between NTI torradoviruses in the RNA1 with Squash chlorotic leaf spot virus (SCLSV), the latest proposed member of the genus, in an independent clade.

CaTV1 - RNA2

RNA2 (4995 nt) has two predicted ORFs encoding two polyproteins. ORF1 encodes a putative polyprotein of 202 aa with a predicted molecular weight of $22 \mathrm{kDa}$ (figure 1). This ORF, partially overlaps the large ORF2, and is a distinguishing feature of the Torradovirus genus. The closest related member was MYMoV (83\% identity). No conserved motifs were found in the sequence and several differences were seen between $\mathrm{TI}$ and NTI torradoviruses, confirming previous results reported [13].

ORF2 encodes a large polyprotein of 1167 aa $(130 \mathrm{kDa})$. The translational start (AUG) codon was found at nt positions 1165-1167 while the stop (UAA) codon was at nt positions 4666-4668. When the whole nucleotide sequence was compared to other members of the Secoviridae family, the most closely related members were LNLCV (68\% identity) and MYMoV (66 \% identity).

The region encoding the movement protein (MP) domain was found between aa 45-239 with the typical LxxPxL motif in positions 211-216. Based on homology to related viruses and previous identification of ToMarV and ToChSV cleavage sites [16], the cleavage site between the MP and the first coat protein is likely to be located at position $487\left(\mathrm{Q}^{487} / \mathrm{A}^{488}\right)$. The coat protein region shares levels of identity of $42.2 \%$ and $47.3 \%$ with LNLCV and MYMoV respectively, which again are the closest related members. This percentage also demarcates CaTV1 as a new species of the genus according to the criteria established in the ICTV ninth report [14]. A glutamine residue (Q) at position 1 is highly conserved in all the torradoviruses and could potentially act as a protease cleavage site among the different proteins [16]. By comparison with ToMarV and ToChSV cleavage sites, a $Q$ residue has been identified at position $695\left(Q^{695} / S^{696}\right)$ which could be a potential cleavage site 
between the Vp35/Vp26 coat proteins. Additionally, the putative Vp26 and Vp23 cleavage site region would be located at aa position $935\left(Q^{935} / I^{936}\right)$. However, the actual number and size of the predicted capsid proteins for CaTV1 have not been determined experimentally.

113 A phylogenetic tree was constructed with all the members of the Torradovirus genus using the whole region with the three coat proteins (Figure $2 b$ ). In agreement with the results obtained with RNA1,

115 three different clades can be again differentiated: five viruses cluster in the tomato-infecting torradovirus clade; CaTV1 is grouped with MYMoV and LNLCV in the non-tomato infecting (NTI)

117 torradovirus clade; and SCLSV clusters with Cassava torrado-like virus (CsTLV) sequences in a third independent clade.

119 CaTV1 is transmitted by aphids [17] and a search of possible aphid transmission motifs was carried out comparing the coat protein region of $\mathrm{TI}$ and NTI torradoviruses. DAG (Asp-Ala-Gly) is usually a highly conserved motif in $\mathrm{N}$-terminal of the coat protein of potyviruses and it has been demonstrated

122 that the change of any of the amino acid prevents transmission by aphids [18]. However this motif could not be found within the CaTV1 sequence. TI torradoviruses have been described to be whiteflytransmitted [19, 20], but no transmission motifs have been identified in the sequences for any of these members so far.

The presence of a small (3-5 kDa) VPg linked to the $5^{\prime}$ end of the RNAs has been confirmed for most members of the order; comparative genomics strongly suggests that this property is universally conserved among the genus and the Secoviridae family [13].

This study has described a complete characterization of the whole genome sequences of CaTV1 for both RNAs by comparison with other members of the genus and confirms that CaTV1 is a new species according to the criteria established by the ICTV. Phylogenetic studies using both RNAs have

132 also confirmed and given further evidence of the differences between non-tomato infecting torradoviruses. 
This research was supported as project FV 382b through UK government funding under Defra Plant Health Capability. All authors declare that they have no conflict of interest. This article does not contain any studies with human participants or animals performed by any of the authors

\section{References}

141 1. Adams I.P., Skelton, A., Macarthur, R., Hodges, T., Hinds, H., Flint, L., Deb Nath, P., Boonham N., 142 Fox. A., (2014). Carrot yellow leaf virus is associated with Carrot Internal Necrosis. PLOSone 9, Issue $143 \quad 11, \mathrm{e} 109125$.

144 2. Rozado-Aguirre, Z., Marais, A., Svanella-Dumas, L, Faure, C., Latour, F., Villeneuve, F., Dickinson, 145 M., Fox, A., Boonham, N., Candresse, T. (2017). First Report of Carrot torradovirus 1 (CaTV1), a 146 member of the torradovirus genus, on carrots in France. Plant disease, http://dx.doi.org/10.1094/PDIS-01-17-0095-PDN

3. Verbeek, M., Dullemans, A.M., van den Heuvel, J.F.J.M., Maris, P.C., van der Vlugt R., (2007). Identification and characterization of tomato torrado virus, a new plant picorna-like virus from tomato. Archives of Virology, 152, 881-890. Tomato marchitez virus, a new plant picorna-like virus from tomato related to tomato torrado virus. Archives of Virology, 153 (1), 127-134. Tomato chocolàte virus: a new plant virus infecting tomato and a proposed member of the genus Torradovirus. Archives of Virology, 155 (5), 751-755 virus, a member of a new torradovirus species that causes a necrosis-associated disease of tomato in Guatemala. Archives of Virology, 155, 857-869. Tomato torrado virus in Poland. Plant Disease, Vol 91, 1364. 
8. Verbeek, M., Dullemans, A., van Raaij, H.M., Verhoeven, J., van der Vlugt, R., (2013). Lettuce necrotic leaf curl virus, a new plant virus infecting lettuce and a proposed member of the genus Torradovirus. Archives of Virology, 159, (4), 801-805.

9. Seo, J.K., Kang, M., Kwak, H.R., Kim M.K., Kim, C.S., Lee, S.H., Kim, J.S., Choi H.S., (2014). Complete genome sequence of motherwort yellow mottle virus, a novel putative member of the genus Torradovirus. Archives of Virology, 160 (2), 587-90.

10. Carvajal-yepes, M., Olaya, C., Lozano, I., Cuervo, M., Castano, M., Cuellar, WJ., (2014). Unraveling complex viral infections in cassava (Manihot esculenta Crantz) from Colombia. Virus Research. 186, 76-86.

172 Characterization and occurrence of squash chlorotic leaf spot virus, a tentative new torradovirus infecting cucurbits in Sudan. Archives of Virology, 161 (6), 1651-1655. Secoviridae: a proposed family of plant viruses within the order Picornavirales that combines the families Sequiviridae and Comoviridae, the unassigned genera Cheravirus and Sadwavirus, and the proposed genus Torradovirus. Archives of Virology, 154 (5), 899-907. Thompson, JR., (2015). Torradoviruses. Annual Review of Phytopathology, Vol 53, 485-512. Committee on Taxonomy of Viruses. San Diego: Elsevier Acad. viruses. J. Gen. Virol, 72 (Pt 9), p. 2197-2206. of the cleavage sites of the RNA2-encoded polyproteins for two members of the genus Torradovirus by $\mathrm{N}$-terminal sequencing of the virion capsid proteins. Virology, Vol 496, 109-115. 

and transmission of Carrot torrado virus, a novel putative member of the Torradovirus genus. Journal of Virological Methods, Vol 235, 119-124. potyvirus transmissibility by aphids. J Gen Virol. 80 (Pt 12), 3281-3288. transmitted in a semi-persistant and stylet-borne manner by different whitefly vector. Virus Research $186,55-60$. (2008). Tomato torrado virus is transmitted by Bemisia tabaci and Infects Pepper and Eggplant in Addition to Tomato. Plant disease, Vol 92 (7), 1139. 
227 Figure 1: Proposed genetic diagram for CaTV1 RNA1 and RNA2 with the positions of the ORFs noted. Relative positions of regions containing helicase, protease and RNA-dependent RNA polymerase motifs on RNA1, and movement protein and coat proteins on RNA2 are indicated.

230

Figure 2: (A) A phylogenetic tree of sequences of viruses within the Secoviridae family was constructed using the RdRp amino acid region. (B) A second phylogenetic tree constructed using the whole coat protein region of CaTV1 RNA2 of all the members of the Torradovirus genus. Alignments were done using MEGA6 using the neighbour-joining algorithm. Sequences of all the viruses were chosen from GenBank and accession numbers are shown. The numbers at the branch points are the percentage bootstrap values following 500 bootstrap resampling and the scale indicates the number of amino acid substitutions per site. 
251 Figure 1

252
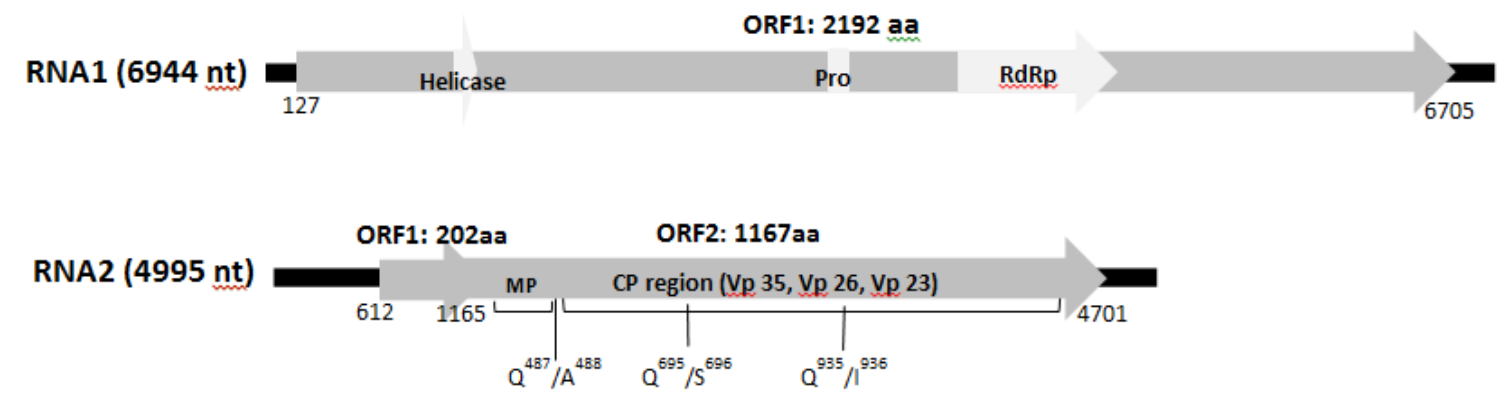

253

254

255

256

257

258

259

260

261

262

263

264

265

266

267 
A
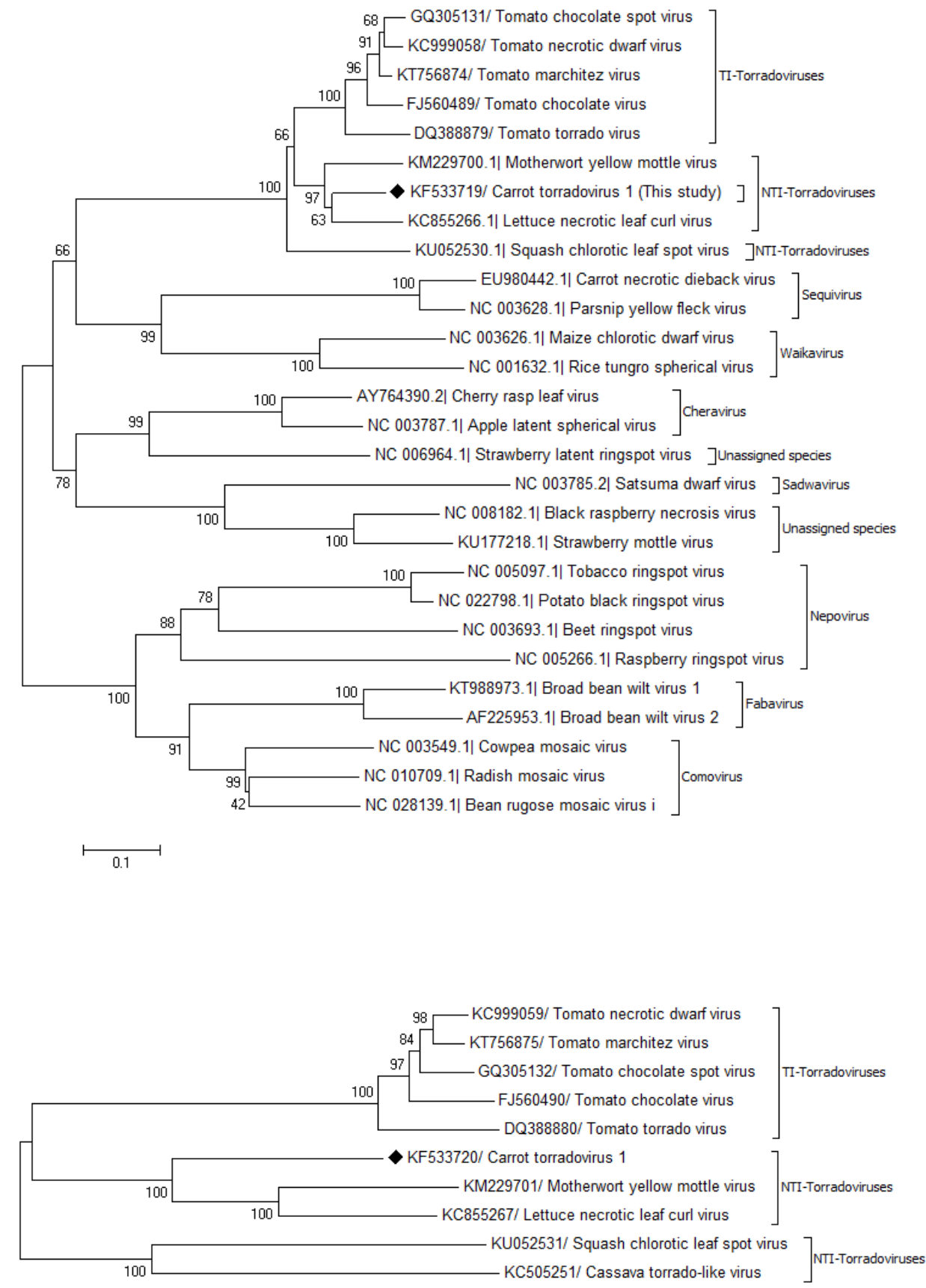